\title{
Field evaluation of Specific Mycobacterial Proteins-Based Skin Test for the Differentiation of Mycobacterium bovis-Infected and Bacillus Calmette Guerin-Vaccinated Crossbred Cattle in Ethiopia
}

\author{
Berecha Bayissa $^{1}$, Aboma Zewude ${ }^{2}$, Adane Worku ${ }^{1}$, Balako Gumi ${ }^{1}$, Stefan Berg ${ }^{3}$, Glyn \\ Hewinson $^{4}$, James Wood ${ }^{5}$, Gareth Jones ${ }^{3}$, Hans Vordermeier ${ }^{6}$, and Gobena Ameni ${ }^{7}$ \\ ${ }^{1}$ Aklilu Lemma Institute of Pathobiology, Addis Ababa University, P.O. Box 1176, Addis \\ Ababa, Ethiopia \\ ${ }^{2}$ Ethiopian Public Health Institute, P.O. Box 1242, Addis Ababa, Ethiopia \\ ${ }^{3}$ Animal and Plant Health Agency \\ ${ }^{4} 5$ Aberystwyth University, Ceredigion SY23 3FL, United Kingdom \\ ${ }^{5}$ University of Cambridge \\ ${ }^{6}$ Animal and Plant Health Agency, New Haw, Addlestone, Surrey, United Kingdom. \\ ${ }^{7}$ Addis Ababa University
}

January 2, 2021

\begin{abstract}
Bovine tuberculosis (bTB) challenges intensive dairy production in Ethiopia and implementation of the test and slaughter control strategy is not economically acceptable in the country. Vaccination of cattle with Bacillus Calmette-Guerin (BCG) could be an important adjunct to control, which would require a diagnostic test to differentiate Mycobacterium bovis (M. bovis)-infected and BCG-vaccinated animals (DIVA role). This study describes evaluation of a DIVA skin test (DST) that is based on a cocktail (DSTc) or fusion (DSTf) of specific (ESAT-6, CFP-10 and Rv3615c) M. bovis proteins in Zebu-Holstein crossbred cattle in Ethiopia. The study animals used were 74 calves (35 BCG-vaccinated and 39 unvaccinated) aged less than three weeks at the start and 68 known bTB positive cows. Six weeks after vaccination, the 74 calves were tested with DSTc and the single intradermal cervical comparative tuberculin (SICCT) test. The cows were tested with DSTc and SICCT test. Reactions to DSTc were not observed in BCG-vaccinated and unvaccinated calves while SICCT test reactions were detected in vaccinated calves. DSTc reactions were detected in $95.6 \%$ of the cows and single intradermal tuberculin (SIT) positive reactions were found in $98.2 \%$ (95\% confidence interval, CI, 92.1-100\%). The sensitivity of DSTc was 95.6\% (95\% CI, 87.6-99.1\%), and significantly $(\mathrm{P}<0.001)$ higher than the sensitivity $(75 \%, 95 \% \mathrm{CI}, 63.0-84.7 \%)$ of the SICCT test at $4 \mathrm{~mm}$ cutoff. DSTf and DSTc reactions were correlated $(\mathrm{r}=0.75 ; 95 \% \mathrm{CI}=0.53-0.88)$. In conclusion, DSTc could differentiate M. bovis-infected from BCG-vaccinated cattle in Ethiopia. DST had higher sensitivity than the SICCT test. Hence, DSTc could be used as a diagnostic tool for bTB if BCG vaccination is implemented for the control of bTB in Ethiopia and other countries.
\end{abstract}

\section{Hosted file}

Berecha et al. 2020-Trans and Emerg.pdf available at https://authorea.com/users/386646/ articles/502100-field-evaluation-of-specific-mycobacterial-proteins-based-skin-testfor-the-differentiation-of-mycobacterium-bovis-infected-and-bacillus-calmette-guerinvaccinated-crossbred-cattle-in-ethiopia 

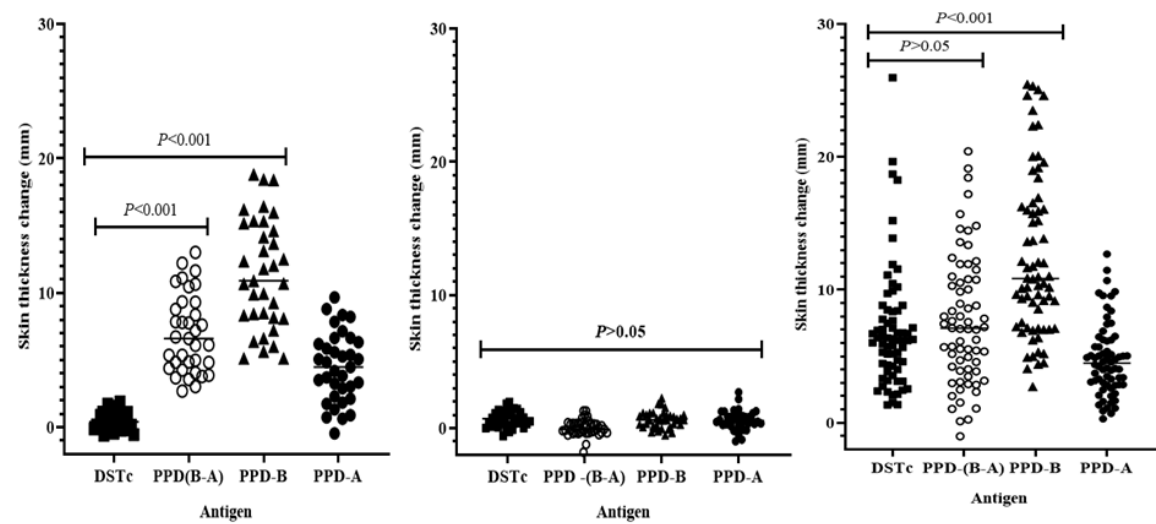

Figure 1.

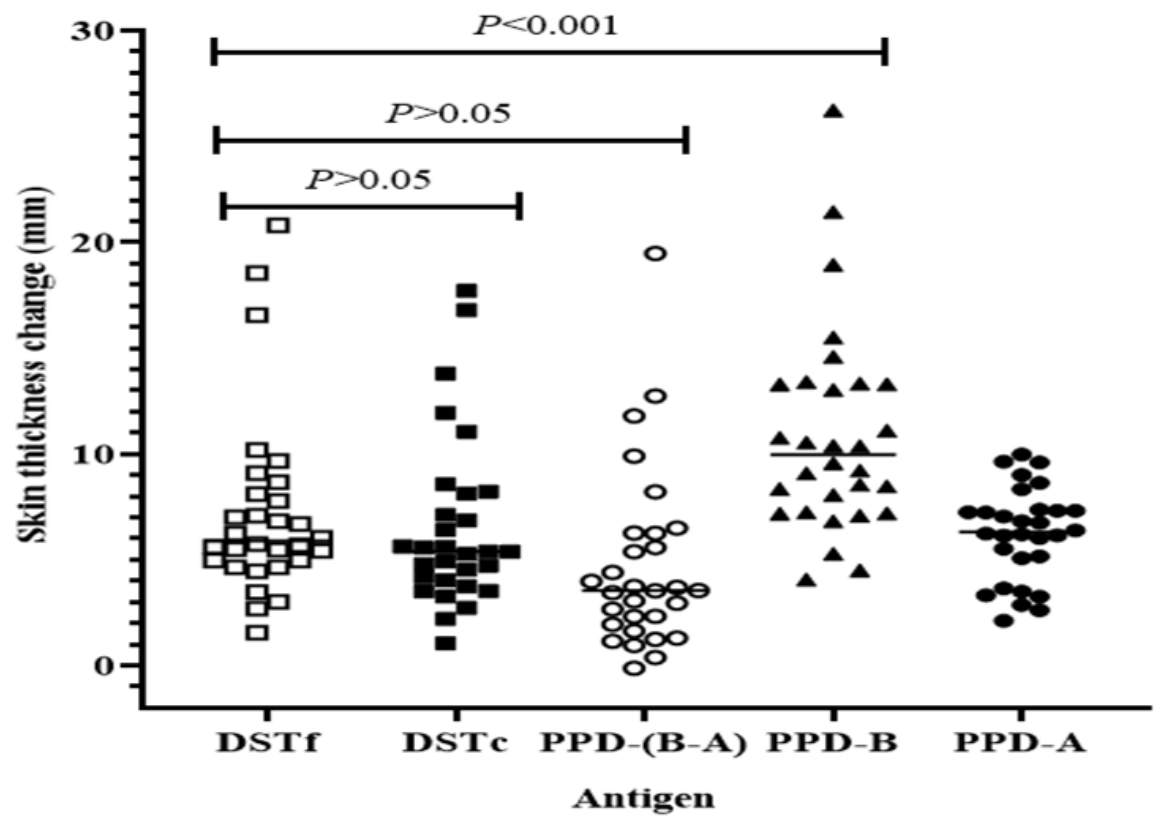

Figure 2 


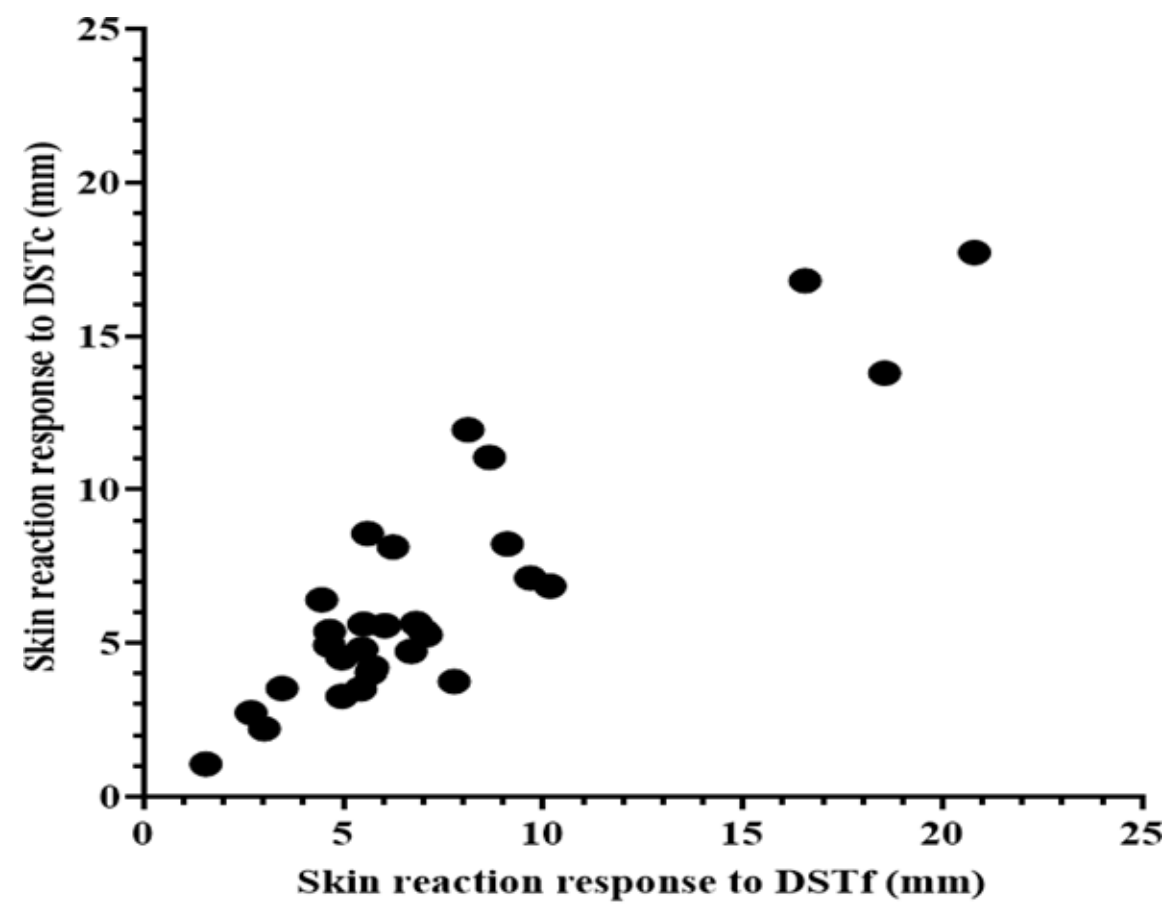

Figure 3

\section{Hosted file}

Table -Trans and Emerging.pdf available at https://authorea.com/users/386646/articles/ 502100-field-evaluation-of-specific-mycobacterial-proteins-based-skin-test-for-thedifferentiation-of-mycobacterium-bovis-infected-and-bacillus-calmette-guerin-vaccinatedcrossbred-cattle-in-ethiopia 